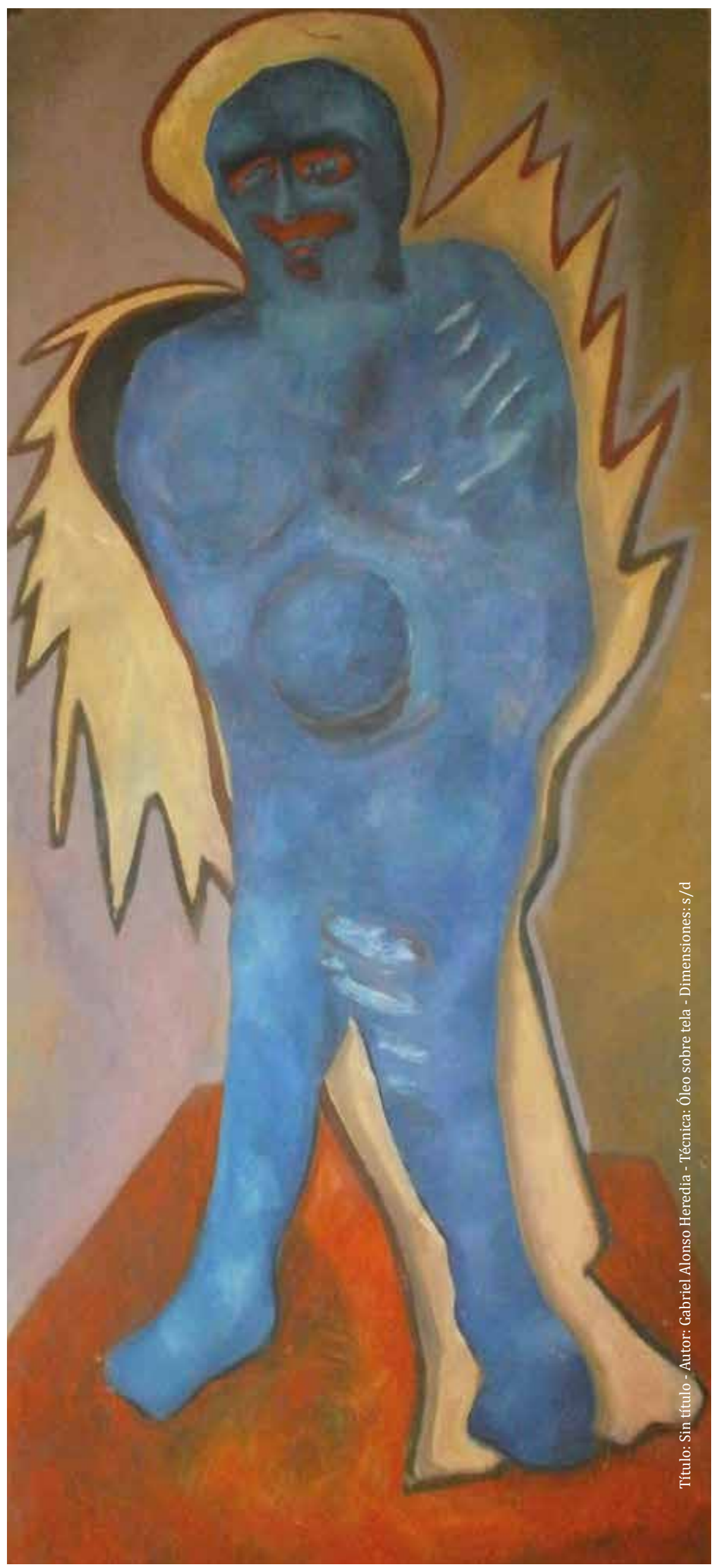


Pedagogía y Saberes No. 48

Universidad Pedagógica Nacional

Facultad de Educación. 2018, pp. 27-41

\title{
Presencia docente
} y construcción de conocimiento en una asignatura universitaria modalidad b-learning

Artículo de investigación

Teaching Presence and Construction of Knowledge in a b-Learning University Course

Presença docente e construção de conhecimento numa matéria universitária na modalidade b-learning

\author{
Nadia Lucía Obando Correal* \\ Andrés Oswaldo Palechor Ocampo ** \\ Diana Marcela Arana Hernández ${ }^{* * *}$
}

Para citar este artículo:

Obando, N., Palechor, A. y Arana, D. (2018). Presencia docente y construcción de conocimiento en una asignatura universitaria modalidad b-learning. Pedagogía y Saberes, 48, 27-41.

* Profesora de la Universidad del Quindío, Colombia. Magíster en Educación de la Universidad Tecnológica de Pereira, Colombia. Investigadora del Grupo BIOEDUQ. Correo electrónico: naluobando@utp.edu.co Código ORCID: orcid.org/0000-0001-9177-4332

** Profesor de la Universidad Tecnológica de Pereira. Colombia. Magíster en Educación de la Universidad Tecnológica de Pereira, Colombia. Investigador del Grupo de Estudios Metodológicos para la Enseñanza de la Matemática y el uso de las Nuevas Tecnologías de la Información y la Comunicación.

Correo electrónico: andrespalechor@utp.edu.co Código ORCID: orcid.org/0000-0003-3034-9804

*** Profesora de la Universidad Tecnológica de Pereira. Colombia. Magíster en Educación y doctoranda en educación de la Universidad Tecnológica de Pereira, Colombia. Investigadora del Grupo Educación y Desarrollo Humano. Correo electrónico: dmarana@utp.edu.co Código ORCID: orcid.org/0000-0003-4359-1720 


\title{
Resumen
}

El artículo presenta los resultados de una investigación que analiza la presencia docente (PD) en una asignatura universitaria con modalidad $b$-learning. El estudio integra un análisis estructural y de contenido para identificar el ejercicio efectivo de la PD y el aporte de cada participante a la construcción del conocimiento. Los resultados muestran que quienes acceden frecuentemente a la plataforma para revisar el foro y hacer contribuciones en diferentes dimensiones y momentos son portadores efectivos de la PD, la cual no sería una característica exclusiva del profesor. De esta manera, el papel que desempeñan las interacciones efectivas en los procesos de aprendizaje y la construcción colaborativa del conocimiento, se considera como el soporte que da sentido a las acciones educativas en las redes asíncronas.

\section{Palabras clave}

presencia docente; redes asíncronas de aprendizaje; influencia educativa; análisis estructural; análisis de contenido

\begin{abstract}
This article presents the results of a research that analyzes teaching presence (TP) in a b-learning university course. The study brings together a structural and a content analysis in order to identify the real exercise of the TP and the contribution of each participant to the construction of knowledge. The results show that those who access the platform frequently to check the forum and make contributions in different dimensions and moments are efficient TP carriers which would not be a teacher-exclusive characteristic. As such, the role of effective interactions in learning processes and the collaborative construction of knowledge are seen as the support that gives sense to educational actions in asynchronous networks.
\end{abstract}

\section{Keywords}

teaching presence; asynchronous learning networks; education influence; structural analysis; content analysis

\section{Resumo}

0 artigo apresenta os resultados de uma pesquisa que analisa a presença docente (PD) em uma matéria universitária na modalidade b-learning. 0 estudo integra uma análise estrutural e de conteúdo para identificar o exercício efetivo da PD e a contribuição de cada participante à construção do conhecimento. Os resultados mostram que aqueles que acessam frequentemente à plataforma para revisar o foro e fazer contribuições em diferentes dimensões e momentos são portadores eficientes da PD, a qual não seria uma característica exclusiva do professor. Portanto, o papel que desempenham as interações efetivas nos processos de aprendizagem e na construção colaborativa do conhecimento, é considerado como o suporte que dá sentido às ações educativas nas redes assíncronas.

\section{Palavras-chave}

presença docente; redes assíncronas de aprendizagem; influência educativa; análise estrutural; análise de conteúdo 


\section{Introducción}

La creación y gestión de nuevos entornos educativos en la actual sociedad digital (Lévy, 2004) ha de atender al potencial transformador que ofrecen las tecnologías de la información y la comunicación (TIC). Al respecto, Bustos (2011) plantea que la aparición de los entornos virtuales de aprendizaje se ha traducido en diversos escenarios para repensar y transformar los contextos educativos (educación presencial) y crear nuevos entornos de enseñanza y aprendizaje (en línea o e-learning, e híbrido o semipresencial) que han sido incorporados a las prácticas educativas en contextos universitarios.

En el Informe Horizon de Educación Superior para América Latina (2013) se expone que los paradigmas de enseñanza están cambiando al incluir la educación en línea, la educación híbrida y los modelos colaborativos en los procesos de enseñanza y aprendizaje. En este sentido, Bustos, Engel y Rocamora (2011) señalan que pese a la diversidad que ofrecen dichos escenarios, existe una tendencia hacia el uso de redes asíncronas de aprendizaje (Asynchronous Learning Networks, ALN) en ambientes educativos universitarios. Esto se debe a que estos espacios de trabajo son propicios para impulsar procesos sustentados en las nociones de aprendizaje colaborativo; además, brindan la posibilidad de participación reflexiva, lo que no ocurre con otras herramientas de comunicación sincrónica, donde la inmediatez supone un obstáculo a la reflexión y el análisis.

Sin embargo, Bustos, Coll y Engel (2009) manifiestan que aún no se terminan de vislumbrar todas las posibilidades de novedad que ofrecen estos escenarios de comunicación asíncrona, entre otras razones porque los avances tecnológicos parecen ir siempre varios pasos por delante de su utilización educativa, además, exigen formas innovadoras en el establecimiento de las relaciones entre los actores educativos. Al respecto, Pérez (2009) señala que en estos entornos virtuales -que han venido en aumento en los últimos años-el rol del docente y de la influencia educativa cobra gran relevancia, pues el maestro será quien reconduzca, haga reflexionar y proponga nuevas orientaciones. De allí la importancia de estudiar la presencia docente en entornos virtuales, entendida como "el conjunto de acciones dirigidas a diseñar, facilitar y orientar los procesos comunicativos y cognitivos de los participantes con el fin de que alcancen unos objetivos de aprendizaje personalmente significativos y educativamente valiosos" (Anderson et al. 2001 citado en Coll, Bustos y Engel, 2009).
Diferentes investigadores, entre ellos Anderson, Rourke y Garrison (2001), Coll, Bustos y Engel (2009), Guilleumas (2015), Borgobello y Roselli (2016), y más recientes, como Coll, Engel y Niño (2017), llaman la atención sobre la necesidad de que el profesor asuma ciertas responsabilidades y desempeñe un papel importante en tales entornos. Asimismo, García y Pineda (2010) reportan la necesidad de indagar sobre el valor agregado que ofrecen las actividades de aprendizaje asíncronas y su contribución a la construcción colaborativa de conocimiento, ya que, aunque estas redes son escenarios idóneos para el fomento de aprendizajes, los resultados de las investigaciones muestran que sus integrantes difícilmente participan en discusiones de alto nivel y sus aportes tienden a ser modestos y responden exclusivamente a lo solicitado por el maestro (Zhu, 2006).

En Colombia, Palacio, Rodríguez y Forero (2015) señalan que la educación en línea o virtual tomó relevancia desde 2010, cuando fue reglamentada por el Decreto 1295 (Ministerio de Educación Nacional). Desde entonces, estos escenarios han hecho emerger nuevas exigencias y roles que permiten implementar mecanismos de comunicación e interacción más amplios y complejos, lo que plantea nuevos retos para docentes y estudiantes. Cuellar Bernal-Velasco, PérezSoto y Montenegro-Aldana (2012) han encontrado que las semejanzas y diferencias en las valoraciones de los estudiantes respecto a la presencia docente está fuertemente influenciada por el papel que este asuma y las ayudas ajustadas que brinda, promoviendo en los estudiantes no solo la participación sino también la colaboración y construcción conjunta.

Por su parte, Tobón, Arbeláez, Falcón y Bedoya (2010) manifiestan que la incorporación de las TIC, particularmente en la Universidad Tecnológica de Pereira, se relaciona directamente con la creación de la Unidad Virtual (Univirtual) en el 2004. En ese año se inició la construcción de asignaturas semipresenciales o blended learning, donde las redes asíncronas o foros académicos constituyen más del $70 \%$ de las actividades usadas en estos entornos (Lozano, 2016). No obstante, las investigaciones sobre su uso adecuado o sobre el desarrollo de procesos para la construcción de conocimiento en dichos ambientes virtuales son escasos e incipientes y no han abordado los procesos de influencia educativa.

Los investigadores citados coinciden en resaltar la importancia de analizar las interacciones que se dan en los entornos educativos virtuales. Esto despierta el interés por indagar específicamente en los foros virtuales académicos que se configuran en el marco de las redes de aprendizaje basadas en la comunicación 
asíncrona escrita, puesto que estos espacios han adquirido un protagonismo destacado, y cada vez más se están configurando como una poderosa herramienta de comunicación y trabajo colaborativo en los ambientes virtuales de aprendizaje, sobre todo en el ámbito de la educación universitaria. Además, el uso de dichos foros ofrece diferentes potencialidades al proporcionar la posibilidad de participación de una forma reflexiva (Coll Bustos y Engel, 2009), frente a otras herramientas de comunicación y trabajo de carácter sincrónico, donde la inmediatez supone un obstáculo a la reflexión y el análisis.

Por lo anterior se plantea como pregunta de investigación: ¿Qué modalidades y grados de presencia docente se encuentran en una secuencia didáctica de una asignatura semipresencial en un contexto de educación universitaria?

\section{Presencia docente e influencia educativa}

El concepto de influencia educativa (Coll, Colomina, Onrubia y Rochera, 1992; Coll, Onrubia y Mauri, 2008; Colomina y Onrubia, 2001) hace referencia a los procesos interpsicológicos mediante los cuales los profesores, y en su caso otros agentes educativos o actores presentes en el contexto de aprendizaje, ayudan a los estudiantes a construir significados progresivamente más ricos, complejos y válidos sobre los contenidos de enseñanza y aprendizaje.

Para que la influencia educativa ejercida por el profesor sea eficaz debe ser contingente y ajustarse al desarrollo y la evolución del proceso de aprendizaje. Así, las ayudas que se entreguen a los estudiantes no pueden ser las mismas, sino que han de ir variando en tipo, cantidad e intensidad en función de los avances y retrocesos que los alumnos experimentan $\mathrm{y}$, a la vez, se deben ir retirando progresivamente a medida que estos se muestran más autónomos en su aprendizaje (Coll, 2010).

El concepto de presencia docente, entendida -desde los mecanismos de influencia educativacomo "el diseño, facilitación y orientación de los procesos cognitivo y social, con el fin de obtener resultados educativos significativos desde el punto de vista personal y docente" (Garrison y Anderson, 2005, p. 51), llama la atención sobre la necesidad de que el profesor asuma ciertas responsabilidades y satisfaga un papel importante en los entornos virtuales de aprendizaje. De esta manera, aunque su implicación en los cursos en línea es fundamental y necesaria, la presencia docente no solo es tarea del profesor; en muchos contextos, especialmente en los universitarios, "es delegada o asumida por estudiantes que contribuyen con sus habilidades y conocimiento al desarrollo de la comunidad de aprendizaje" (Anderson, 2004, p. 274).
Se propone por ello una responsabilidad y un control compartidos de las funciones, de manera que se establezca un desafío que permita trabajar las ideas falsas, el conflicto cognitivo y un discurso dirigido hacia los niveles más altos del aprendizaje: reflexivo, profundo y significativo.

Para Bustos (2011), estos enfoques planteados por Garrison y Anderson (2005) reconocen que el aprendizaje de los alumnos es producto del proceso de construcción personal y de la dinámica del conjunto de relaciones que se establecen entre los actores del proceso educativo. Así, se espera que en las comunidades de aprendizaje virtuales la responsabilidad y el control evolucionen de forma natural permitiendo que la presencia docente se vuelva más distribuida.

Conviene por lo tanto no confundir la presencia docente con la presencia del docente, siendo la segunda un caso particular e importante, pero consecuente de la primera. Para ello, Bustos et al. (2009) aclaran:

1. El hecho de que el profesor esté presente (presencia del docente) no garantiza que esté ejerciendo una presencia docente entendida como el ejercicio de una influencia educativa eficaz.

2. La presencia docente, desde la perspectiva de influencia educativa, puede ser ejercida en primera instancia por el profesor, pero también por otros participantes; de aquí la noción de Presencia Docente Distribuida (Garrison y Anderson, 2005).

\section{Redes asíncronas y presencia docente en la educación universitaria}

Como ya se ha planteado, los foros virtuales de aprendizaje, en el marco de las ALN, constituyen uno de los escenarios de mayor uso en procesos educativos universitarios mediados por TIC (Coll, 2009; Coll, Bustos y Engel, 2009; Coll, Engel y Niño, 2017; Cuéllar, Bernal-Velasco, Pérez-Soto y MontenegroAldana, 2012; Guilleumas, 2015); sin embargo, los estudios frente a la presencia docente siguen siendo incipientes. Al respecto, Cuellar et al. (2012) reportan que los estudiantes universitarios que desarrollan actividades de aprendizaje en estos espacios virtuales se ven influenciados significativamente en los procesos de construcción de conocimiento por el papel que el docente asuma y las ayudas ajustadas que brinda, de manera que promueve en los estudiantes la participación, la colaboración y la construcción conjunta.

Por su parte, Hernández-Sellés, GonzálezSanmamedy y Muñoz-Carril (2015) afirman que uno de los desafíos a los que se enfrentan las instituciones de educación superior desde estos ambientes es la adopción de nuevos modelos articulados en torno a la gestión del conocimiento desde la visión de las ecologías de aprendizaje, donde se fomente una 
distribución de responsabilidades entre docentes y alumnos que permita el empoderamiento de los individuos, basado en el tratamiento mutuo como personas maduras y autónomas, de tal manera que haya una transferencia real de saberes entre los diversos participantes de estas comunidades.

En este sentido, para Coll et al. (2017), los aspectos estructurales (acceso, participación y conectividad) y de contenido (gestión de la participación, de la tarea y de los significados) son indicadores vitales para analizar la forma en que los participantes de los foros en línea contribuyen y aportan a la construcción de conocimiento. Esto, debido a que bajo el modelo de influencia educativa distribuida, y en situaciones de trabajo y aprendizaje colaborativo, todos los participantes son fuentes potenciales de ayuda para los otros participantes.

Teniendo en cuenta los planteamientos presentados hasta el momento, se propone centrar la atención en el análisis de la presencia docente, reconociendo la relevancia de la acción dialógica entre los participantes como aspecto central para la enseñanza y el aprendizaje de conceptos de alto nivel (Bustos, 2011). Desde la perspectiva de la influencia educativa, la responsabilidad y el control del aprendizaje pueden pasar del profesor al estudiante, en la medida en que a estos se les permita progresivamente ser más autónomos, con ajustes continuos a las ayudas prestadas, que se relacionan directamente con los avances y retrocesos, incluso de quienes entregan dichas ayudas.

En el caso de las redes asíncronas de aprendizaje o foros de discusión, la posibilidad de ofrecer y recibir ayudas está condicionada por el cumplimiento de ciertas exigencias. Así, es necesario que los participantes revisen (lean) y realicen (escriban) contribuciones y aportes a los foros con cierta frecuencia y periodicidad. Es prácticamente imposible que aquellos cuya implicación en la actividad conjunta no cumpla los requisitos mencionados sean portadores de presencia docente y puedan proporcionar ayudas ajustadas y útiles al resto de participantes (Bustos, 2011).

\section{Metodología}

La investigación tiene un enfoque interpretativo. Es un estudio de caso ya que se pretende analizar y comprender las particularidades y complejidades de una situación específica (Stake, 2005); se analizan, desde la perspectiva de la influencia educativa, las modalidades y grados de presencia docente desplegados por los participantes de una red asíncrona de aprendizaje desarrollada en un foro de discusión.

\section{Contexto de observación y participantes}

El escenario es Pedagogía en Ambientes Virtuales de Aprendizaje, una asignatura electiva de modalidad semipresencial desarrollada en la plataforma Moodle, que se ofrece a los diversos programas de licenciatura de la Universidad Tecnológica de Pereira. El curso está conformado por 19 estudiantes ( 9 hombres y 10 mujeres) y una docente, quienes accedieron voluntariamente a participar en la experiencia investigativa luego de firmar un consentimiento informado.

La unidad de observación, análisis e interpretación corresponde a una secuencia didáctica completa (SD) (Camps, 2003) desarrollada en una red asíncrona de aprendizaje, específicamente a través de un foro de debate sencillo ${ }^{1}$. La SD tiene una duración de 15 días, tiempo en el cual los participantes analizan y discuten el modelo pedagógico idóneo para implementar en un ambiente virtual de aprendizaje. El tema del foro se desarrolla teniendo como referentes dos recursos: el artículo "Aprender y enseñar en entornos virtuales: actividad conjunta, ayuda pedagógica y construcción de conocimiento" (Onrubia, 2005) y el video "Conectivismo y aprendizaje en red" (Leal, 2011). La docente brinda las indicaciones (pedagógicas y tecnológicas), que incluyen la realización de mínimo tres aportes en diferentes periodos y de acuerdo a la rúbrica TIGRE$^{2}$ (Galvis, 2005), que constituye un instrumento de evaluación.

\section{Recolección y análisis de datos}

El cuerpo principal de datos corresponde a los registros en la plataforma Moodle, a las actividades realizadas en ella y al conjunto de contribuciones del profesor y los estudiantes. Su análisis se hace desde una propuesta multimétodo (Bustos 2011; Coll, Bustos y Engel, 2009), e incorpora dos vías de análisis complementarias (estructural y de contenido), que se explican a continuación.

1 Foro donde el docente propone un tema y todos los estudiantes pueden intervenir en él.

2 Acrónimo que identifica las características de los aportes en línea para discusiones pragmáticas: título diciente, ilación, generación de discusión, redacción y presentación, y enriquecimiento de la discusión. 


\section{Análisis estructural}

Tiene en cuenta dos dimensiones que brindan información frente a qué hacen los participantes, cuándo lo hacen, cómo participan y con quién interactúan. Para la primera dimensión, la de acceso y participación, el recurso primario es el registro de actividad que proporciona Moodle, el cual se evalúa a través de un conjunto de indicadores que dan cuenta de lo que hacen los participantes y cuya frecuencia o modalidad es susceptible de ser relacionada desde un punto de vista teórico con un eventual ejercicio de la presencia docente. Para la segunda dimensión, la de conectividad, se utiliza el software NodexL, que permite hacer una representación visual de la red de conexiones entre los participantes.

Para cada dimensión, los índices presentan un valor umbral por encima del cual la presencia de los participantes es teóricamente propicia, para el ejercicio de la presencia docente (en la tabla 1 se muestran los índices e indicadores y valores idóneos).

\section{Análisis del contenido}

Se realiza mediante tres dimensiones (véase la tabla 2) con el fin de determinar, a través de las contribuciones de los participantes, de qué hablan, cómo hablan y para qué lo hacen. Su objetivo es identificar y analizar las ayudas que se proporcionan a los participantes, su mayor o menor grado de ajuste a las necesidades que experimentan y su evolución en el transcurso de las actividades de aprendizaje, así como los patrones interactivos y comunicativos y los recursos semióticos utilizados para ello. Con el fin de examinar el contenido se define la unidad de análisis (el fragmento como unidad de codificación), la construcción del sistema de categorías y el procedimiento o protocolo para el análisis, que se realiza con el apoyo del software Atlas.ti.

Tabla 1. Índices e indicadores utilizados para el análisis estructural (acceso, participación y conectividad) y perfil potencial idóneo para el ejercicio de la presencia docente

\begin{tabular}{|c|c|c|}
\hline \multirow{6}{*}{$\begin{array}{l}\text { Dimensión } \\
\text { de acceso y } \\
\text { participación }\end{array}$} & Indices e indicadores & Valor idóneo \\
\hline & $\begin{array}{l}\text { Índice individual de acceso } \\
\text { Total de días de acceso del participante/total de días de } \\
\text { duración de la actividad }\end{array}$ & $\geq 0,5$ \\
\hline & $\begin{array}{l}\text { Patrón individual de acceso } \\
\text { Continuo: ningún periodo con } 5 \text { o más días sin acceso o no más } \\
\text { de tres periodos de } 3 \text { días sin acceso } \\
\text { Discontinuo: uno o más periodos de } 5 \text { o más días sin acceso o } \\
\text { más de tres periodos de } 3 \text { días sin acceso }\end{array}$ & Continuo \\
\hline & $\begin{array}{l}\text { Índice individual de lectura } \\
\text { Total de contribuciones leídas por el participante/total de } \\
\text { contribuciones realizadas por los otros participantes }\end{array}$ & $\geq 0,8$ \\
\hline & $\begin{array}{l}\text { Índice individual de contribuciones } \\
\text { Número total de contribuciones realizadas por el participante/ } \\
\text { número total de contribuciones requeridas a cada participante }\end{array}$ & $\geq 1,4$ \\
\hline & $\begin{array}{l}\text { Patrón individual de contribuciones } \\
\text { Frecuencia de las contribuciones del participante -alta, media, } \\
\text { baja- en cada uno de los tres periodos (inicial, intermedio, final) } \\
\text { Estable: (A, M, B) } \\
\text { No estable: ascendente (B-M-A), descendente (A-M-B), } \\
\text { indefinido (por ejemplo, M-B-A) }\end{array}$ & $\begin{array}{l}\text { Estable (M o A) } \\
\text { No estable ascendente (con } \\
\text { un periodo B como máximo) } \\
\text { No estable descen- } \\
\text { dente (con un periodo } \\
\text { B como máximo) }\end{array}$ \\
\hline
\end{tabular}




\begin{tabular}{|c|c|c|}
\hline \multirow{3}{*}{$\begin{array}{l}\text { Dimensión de } \\
\text { conectividad }\end{array}$} & $\begin{array}{l}\text { Índice de emisión de respuestas } \\
\text { Número total de respuestas que cada participante escribe } \\
\text { al resto del grupo/total de mensajes escritos por } \\
\text { dicho participante. }\end{array}$ & $\geq 0,5$ \\
\hline & $\begin{array}{l}\text { Índice individual de reciprocidad } \\
\text { Número de díadas recíprocas diferentes de las que forma parte } \\
\text { un participante en una red/número total de díadas recíprocas } \\
\text { posibles de las que podría formar parte en dicha red. }\end{array}$ & $\geq 0,5$ \\
\hline & $\begin{array}{l}\text { Índice individual de responsividad } \\
\text { Número de díadas responsivas diferentes de las que forma parte } \\
\text { un participante en una red/número total de díadas responsivas } \\
\text { posibles de las que podría formar parte en dicha red. }\end{array}$ & $\geq 0,5$ \\
\hline
\end{tabular}

Fuente: elaboración propia con base en Bustos (2011)

Finalmente, se relacionan los resultados del análisis estructural y de contenido desde una perspectiva de complementariedad, para identificar tendencias de perfiles de los participantes respecto a la presencia docente distribuida durante la SD. Bustos (2011) plantea que los aspectos concernientes a los requisitos de la participación y la relevancia de las contribuciones de los participantes para apoyar el proceso de construcción de significados y atribución de sentido constituyen el núcleo del modelo de análisis de la presencia docente en redes asíncronas de aprendizaje.

Tabla 2. Dimensiones utilizadas en el análisis de las contribuciones de los participantes

\begin{tabular}{|l|l|}
\hline \multicolumn{1}{|c|}{ Dimensiones } & \multicolumn{1}{c|}{ Aspectos relacionados } \\
\hline Gestión de la participación social (GPS) & $\begin{array}{l}\text { Quién puede hacer o decir qué, cuándo, cómo, } \\
\text { dirigiéndose a quién, con qué medios }\end{array}$ \\
\hline Gestión de la tarea académica (GTA) & $\begin{array}{l}\text { Qué hay que hacer, cómo hay que hacerlo, mediante } \\
\text { qué procedimiento(s), qué producto(s) hay que } \\
\text { generar, con qué características }\end{array}$ \\
\hline $\begin{array}{l}\text { Gestión de significados relativos a los } \\
\text { contenidos de aprendizaje (GS) }\end{array}$ & $\begin{array}{l}\text { Presentación, confrontación, negociación y } \\
\text { construcción de significados compartidos } \\
\text { sobre el contenido de aprendizaje }\end{array}$ \\
\hline
\end{tabular}

Fuente: Bustos (2011)

En la tabla 3 se presentan las tres dimensiones junto con una descripción del sistema de categorías de análisis asociadas a ellas y sus códigos respectivos.
Cabe señalar que las categorías emergentes en el presente estudio guardan estrecha relación con las presentadas por Bustos (2011). 
Tabla 3. Dimensiones y categorías emergentes utilizadas en el análisis de las contribuciones de los participantes

\begin{tabular}{|c|c|}
\hline Dimensiones & Categorías \\
\hline \multirow{3}{*}{$\begin{array}{l}\text { Gestión de la } \\
\text { participación social }\end{array}$} & Formulación de pautas de participación FR \\
\hline & Formulación de precisiones sobre las pautas de participación FP \\
\hline & Valoración del grado de cumplimiento de las pautas de participación vc \\
\hline \multirow{6}{*}{$\begin{array}{l}\text { Gestión de la tarea } \\
\text { académica }\end{array}$} & $\begin{array}{l}\text { Formulación de las características de la tarea, su abordaje y su producto } \\
\text { o resultado FR }\end{array}$ \\
\hline & $\begin{array}{l}\text { Petición o exigencia de precisiones sobre las características o exigencias } \\
\text { de la tarea, su abordaje o producto PP }\end{array}$ \\
\hline & $\begin{array}{l}\text { Formulación de precisiones sobre las características o exigencias de la tarea, } \\
\text { por requerimiento de otros participantes FP }\end{array}$ \\
\hline & $\begin{array}{l}\text { Valoración de las características o exigencias de la tarea, su abordaje y su } \\
\text { producto o resultado VE }\end{array}$ \\
\hline & $\begin{array}{l}\text { Valoración del grado de respeto o cumplimiento de las exigencias de la tarea, } \\
\text { su abordaje y su producto o resultado vc }\end{array}$ \\
\hline & $\begin{array}{l}\text { Propuesta de revisión o reformulación de las características o exigencias de la tarea, } \\
\text { su abordaje y su producto o resultado PR }\end{array}$ \\
\hline \multirow{12}{*}{$\begin{array}{l}\text { Gestión de significados } \\
\text { relativos a los contenidos } \\
\text { de aprendizaje }\end{array}$} & Aportación a iniciativa propia de significados propios o presentados como propios SP \\
\hline & Aportación a iniciativa propia de significados atribuidos a fuentes externas SF \\
\hline & Referencia a una o más fuentes de significados (libros, artículos, páginas web, etc.) RF \\
\hline & $\begin{array}{l}\text { Referencia a significados atribuidos al autor del texto mediante cita literal } \\
\text { entrecomillada o con referencia exacta de página o capítulo RFM }\end{array}$ \\
\hline & Incorporación de significados mediante adjuntos de autoría propia o ajena Doc \\
\hline & $\begin{array}{l}\text { Recordatorio literal o casi literal de significados presentados previamente por } \\
\text { otros participantes RE }\end{array}$ \\
\hline & Valoración favorable de significados aportados previamente por otros participantes VF \\
\hline & Valoración crítica de significados aportados previamente por otros participantes vc \\
\hline & Identificación de tópicos o temas de atención, indagación y discusión IT \\
\hline & $\begin{array}{l}\text { Identificación y/o corrección de errores, incomprensiones o lagunas (verdaderos } \\
\text { o no) en los significados aportados previamente por otros o por uno mismo IE }\end{array}$ \\
\hline & $\begin{array}{l}\text { Expresión o manifestación de dudas, interrogantes o incomprensiones respecto } \\
\text { a uno o varios de los tópicos de discusión ED }\end{array}$ \\
\hline & $\begin{array}{l}\text { Formulación de síntesis, resúmenes o recapitulaciones integrando los significados } \\
\text { aportados previamente por uno mismo y por otros participantes SI }\end{array}$ \\
\hline
\end{tabular}

Fuente: Bustos (2011) 


\section{Resultados y discusión}

\section{Perfiles de presencia}

\section{y conectividad: análisis estructural}

\section{Perfiles de presencia: dimensiones de acceso y participación}

Los perfiles se ordenan y agrupan en función de su proximidad al perfil teóricamente idóneo para el ejercicio de la presencia docente, teniendo en cuenta los factores asociados a las dimensiones de acceso y participación. La frecuencia de acceso se relaciona con la posibilidad de seguir el hilo de las contribuciones y, por tanto, ofrecer ayudas al resto de participantes. El patrón de acceso se relaciona con la complejidad que supondría ejercer una influencia educativa en un marco de presencia más bien discontinua. La lectura de las contribuciones de los otros permite compartir el contexto discursivo que se está construyendo y, con ello, ofrecer ayuda. Las contribuciones mínimas esperadas permiten participar ofreciendo ayuda a los otros. Y finalmente, el patrón de contribuciones se relaciona con la necesidad de generar cierta estabilidad a lo largo de las fases de la actividad (Bustos, 2011).

En la siguiente tabla se presentan los resultados del análisis estructural. Aquí los participantes se organizan en cinco grupos, de acuerdo a su proximidad al perfil idóneo para el ejercicio de la presencia docente.

Tabla 4. Perfiles estructurales de presencia docente ordenados de acuerdo a su proximidad al perfil idóneo para el ejercicio de la presencia docente

\begin{tabular}{|c|c|c|c|c|c|c|}
\hline $\begin{array}{l}\text { Grupo } \\
\text { (Cumplimiento } \\
\text { de criterios) }\end{array}$ & $\begin{array}{c}\text { P } \\
\text { Participante }\end{array}$ & $\begin{array}{c}\text { IIA } \\
\text { Índice } \\
\text { individual } \\
\text { de acceso }\end{array}$ & $\begin{array}{c}\text { PIA } \\
\text { Patrón } \\
\text { individual } \\
\text { de acceso }\end{array}$ & $\begin{array}{c}\text { IIL } \\
\text { Indice } \\
\text { individual } \\
\text { de lectura }\end{array}$ & $\begin{array}{c}\text { IIC } \\
\text { Indice } \\
\text { individual de } \\
\text { contribuciones }\end{array}$ & $\begin{array}{c}\text { PIC } \\
\text { Patrón individual } \\
\text { de contribuciones }\end{array}$ \\
\hline \multirow{3}{*}{$\begin{array}{l}\text { Grupo } 1 \\
\begin{array}{l}(504 \\
\text { criterios })\end{array}\end{array}$} & $\mathrm{E} 4$ & 0,9 & continuo & 0,5 & 1,7 & $\begin{array}{c}\text { no estable } \\
\text { descendente }\end{array}$ \\
\hline & $\mathrm{P}$ & 0,7 & continuo & 1,0 & 4,0 & estable medio \\
\hline & E3 & 0,7 & continuo & 0,6 & 1,7 & no estable ascendente \\
\hline $\begin{array}{l}\text { Grupo } 2 \\
\text { ( } 3 \text { criterios) }\end{array}$ & E5 & 0,4 & continuo & 0,4 & 1,7 & $\begin{array}{c}\text { no estable } \\
\text { descendente }\end{array}$ \\
\hline \multirow{2}{*}{$\begin{array}{l}\text { Grupo } 3 \\
\text { (2 criterios) }\end{array}$} & $\mathrm{E} 2$ & 0,6 & continuo & 0,2 & 1,0 & no estable/indefinido \\
\hline & E16 & 0,5 & continuo & 0,3 & 1,0 & no estable/indefinido \\
\hline \multirow{3}{*}{$\begin{array}{l}\text { Grupo } 4 \\
\text { (1 criterio) }\end{array}$} & E18 & 0,4 & continuo & 0,3 & 1,3 & bajo estable \\
\hline & $\mathrm{E} 17$ & 0,4 & continuo & 0,2 & 1,0 & no estable/indefinido \\
\hline & E14 & 0,3 & continuo & 0,3 & 1,0 & no estable/indefinido \\
\hline \multirow{11}{*}{$\begin{array}{c}\text { Grupo } 5 \\
\text { (0 criterios) }\end{array}$} & E6 & 0,2 & discontinuo & 0,3 & 1,3 & no estable/indefinido \\
\hline & E15 & 0,2 & discontinuo & 1,0 & 1,3 & no estable/indefinido \\
\hline & E10 & 0,2 & discontinuo & 0,2 & 1,0 & no estable/indefinido \\
\hline & E19 & 0,2 & discontinuo & 0,1 & 1,0 & no estable/indefinido \\
\hline & E9 & 0,2 & discontinuo & 0,1 & 0,3 & no estable/indefinido \\
\hline & E11 & 0,1 & discontinuo & 0,2 & 0,7 & no estable/indefinido \\
\hline & E13 & 0,1 & discontinuo & 0,1 & 1,0 & no estable/indefinido \\
\hline & E8 & 0,1 & discontinuo & 0,1 & 0,7 & no estable/indefinido \\
\hline & E7 & 0,1 & discontinuo & 0,1 & 0,3 & no estable/indefinido \\
\hline & $\mathrm{E} 12$ & 0,1 & discontinuo & 0,1 & 0,3 & no estable/indefinido \\
\hline & E1 & 0,1 & discontinuo & 0,0 & 0,0 & no estable/indefinido \\
\hline
\end{tabular}

Fuente: Datos obtenidos del corpus documental 
Los resultados del análisis estructural muestran la distribución del ejercicio potencial de la presencia docente y permiten: a) identificar un conjunto de participantes como portadores potenciales de presencia docente (grupos 1 y 2), b) descartar un conjunto de participantes cuyo perfil se aleja del idóneo (grupos 4 y 5), y c) identificar participantes que por su perfil de participación son susceptibles de ser portadores potenciales de modalidades distintas de ejercicio de presencia docente y que, en consecuencia, deben ser foco de atención en el análisis de contenido (grupo 3).

\section{Perfiles estructurales: dimensión de conectividad}

Como lo muestra la figura 1 , la dimensión de conectividad permite observar las relaciones que los participantes establecen entre sí, tales como la cohesión, la centralidad y la densidad de participación a través de los diferentes índices. Aspectos como la emisión de respuestas evidencian que todos, excepto uno de los participantes, contribuyen en mayor o menor medida realizando al menos una contribución dirigida al grupo como respuesta o réplica, bien sea de la semilla de discusión o de otro comentario realizado por un participante. No obstante, aspectos como la reciprocidad y la responsividad no alcanzan los valores idóneos, puesto que no se establecen díadas recíprocas o responsivas de manera significativa en los estudiantes; el único participante que lo hace es el profesor, que se convierte así en el centro de la red.

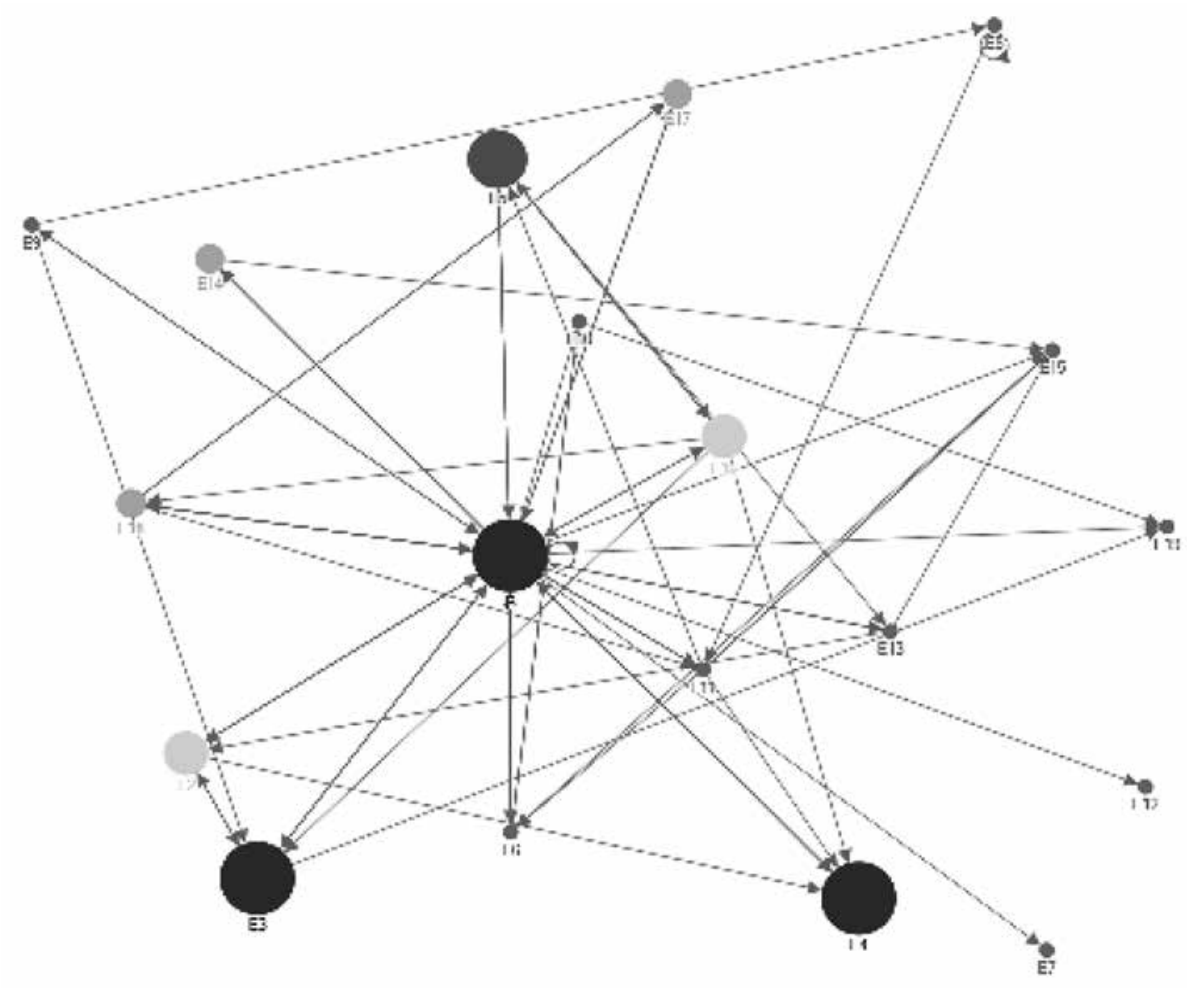

Figura 1. Red de conexiones entre los participantes (dimensión de conectividad). 


\section{Análisis de contenido}

El cuerpo principal de datos para el análisis de contenido es el número total de contribuciones escritas (70) por los 20 participantes. Durante el proceso de codificación se identificaron 247 fragmentos asociados a las tres dimensiones de la presencia docente (Gestión de la participación social, GPS), Gestión de la tarea académica, GTA, y gestión de los significados, GS), los cuales se observan en el gráfico 1 .

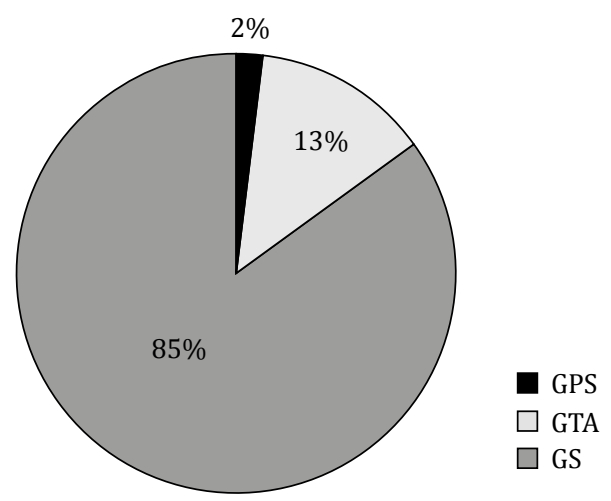

Gráfico 1. Frecuencia y porcentaje de fragmentos codificados en las tres dimensiones de la presencia docente

Fuente: elaboración propia

De esta manera, es posible identificar el discurso que se construye en el aula, el cual evidencia el ejercicio de la influencia educativa en la medida en que los participantes utilizan estrategias discursivas para construir conocimiento en colaboración, utilizando como excusa los contenidos escolares. Así, el hecho de que el $85 \%$ de los fragmentos categorizados correspondan a la gestión de significados y constituyan el eje central del proceso representa aspectos significativos en la apropiación de conocimiento, ya que como lo plantea Onrubia (1997), aprender, "implica fundamentalmente elaborar y reelaborar 'significados' sobre aspectos o parcelas específicas de la realidad, y no simplemente reproducir de manera inmediata elementos de esa parcela de la realidad" (p. 16).

Aunque la gestión de la tarea académica y de participación social también es un aspecto importante por tener en cuenta, el hecho de construir un discurso de naturaleza reflexiva exige una actitud comprometida con el proceso. Mantener esa actitud y promover las aportaciones de calidad requiere que el discurso esté bien centrado y que sea productivo, aspectos que se develan a través de la gestión de significados. En este sentido, otros estudios que indagan sobre el uso de los foros virtuales para la construcción de conocimiento (Gros y Adrián, 2009; Gros y García González, 2013) en diversos escenarios de educación superior han encontrado que la mayor parte de los temas de discusión se sitúan sobre problemas relativos a los contenidos trabajados en clase, aportaciones relativas al funcionamiento de la clase o a la resolución de algún tipo de dudas o problemas, planteados por el profesor. Llama la atención que en este escenario, este no sea el caso.

\section{La gestión de los significados}

Como se observa en el gráfico 2, los participantes que realizan un mayor número de aportes y que además lo hacen en el abanico más amplio de categorías (véase la tabla 3) corresponden al grupo 1: el profesor (P) presenta fragmentos codificados en 11 de las 12 categorías; E4 en 9 de las 12 categorías y E3 en 8.

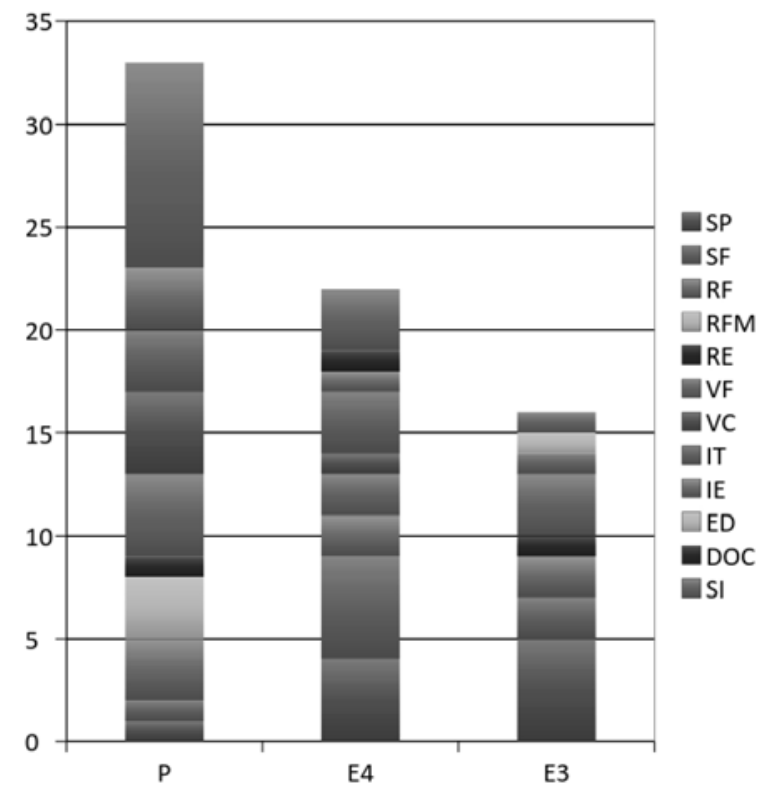

Gráfico 2. Codificación de las contribuciones de los participantes con relación a la gestión de significados (grupo 1).

Fuente: elaboración propia

Por el contrario, tal y como se aprecia en el gráfico 3, el grupo 5 presenta un menor número y variedad de categorías, identificadas a través de sus contribuciones. 


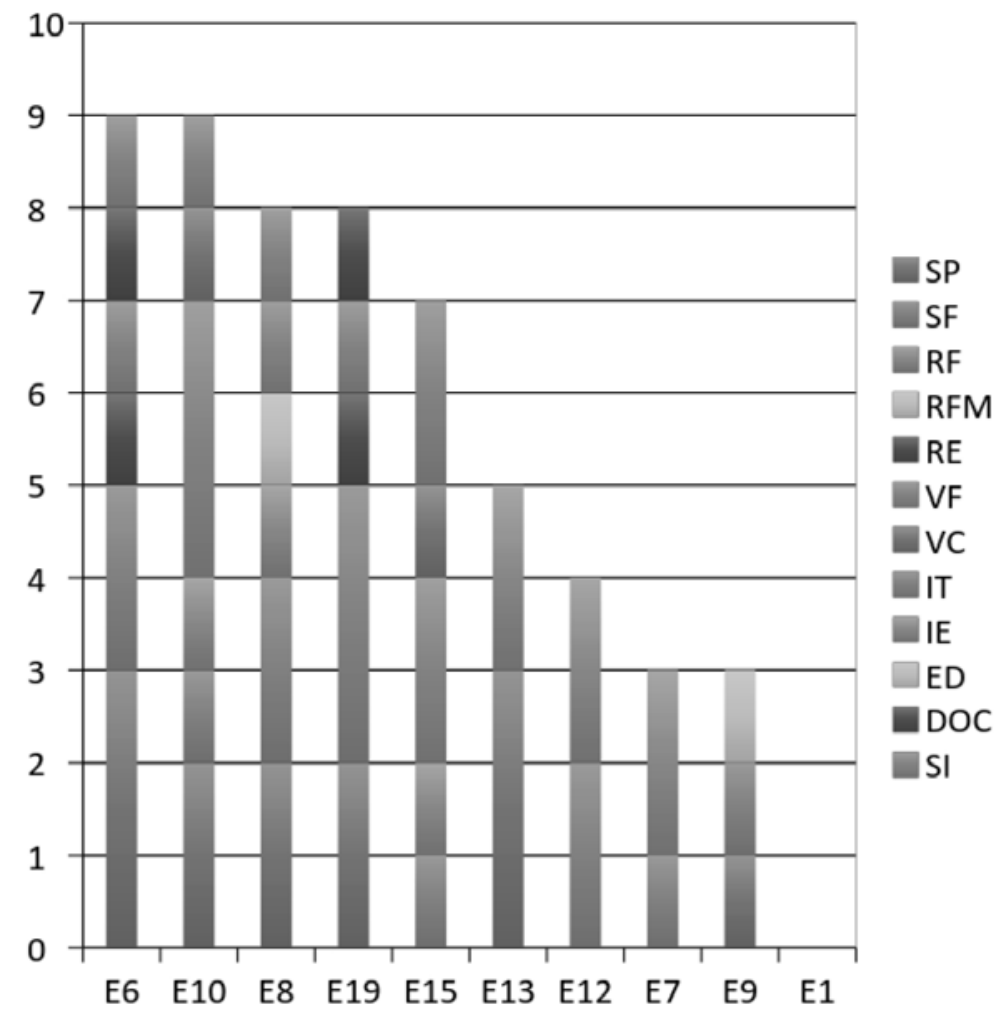

Gráfico 3. Codificación de las contribuciones de los participantes con relación a la gestión de significados (grupo 5).

Fuente: elaboración propia

El ejercicio real de la presencia docente es mayor cuanto más próximo es el perfil de los participantes al perfil idóneo para el ejercicio de la presencia docente: grupo 1 y grupo 2 . Asimismo, estos participantes hacen aportaciones que abarcan un rango más amplio de categorías (gestión de significados) y con fragmentos asociados a las categorías consideradas como relevantes para los procesos de negociación de significados.

\section{La gestión de la tarea académica y la participación social}

La tarea académica y la participación social son las dimensiones que presentan fragmentos asociados en un $13 \%$ y un $2 \%$ respectivamente. Estos sucesos se pueden entender desde el propio proceso de planeación, ya que la docente no promueve procesos de participación social de forma explícita para el desarrollo de la actividad.

\section{Modalidades de ejercicio de la presencia docente}

Mediante los análisis realizados se identifican diversas modalidades y grados de presencia docente desplegados por los participantes del caso estudiado (véase la figura 2). Se encuentra una modalidad denominada Gestión de la presencia docente, a la que se le atribuye un grado total puesto que se da como resultado de un perfil estructural idóneo y de un análisis de contenido idóneo representado en la intersección de las tres gestiones estudiadas (tarea académica, participación social y significados). Bajo esta modalidad y grado se identifica a P, como portador efectivo de presencia docente.

También se identifican participantes que presentan perfiles estructurales idóneos y ejercen presencia docente en algunas de las gestiones, por lo que se ha denominado con un grado parcial. En este sentido, E4 ejerce presencia docente parcial en las modalidades de Gestión de significados y de la tarea académica 
y E3 lo hace respecto a Gestión de significados. Así también, E5 es portador de presencia docente en la modalidad de gestión de significados.

Por otro lado, y a pesar de no cumplir o tener perfiles estructurales idóneos, desde el análisis de contenido presentan fragmentos distribuidos de forma significativa en el tiempo y en las diversas categorías, para alguna de las gestiones, por lo que se les ha denominado portadores de determinada modalidad con un grado focalizado. Por ejemplo, E16, de G3, constituye un caso particular de este tipo al presentar un perfil estructural que lo descarta para ser portador efectivo de presencia docente; no obstante, en sus aportaciones se destacan las contribuciones realizadas en la gestión de significados, presentando fragmentos distribuidos en más del $50 \%$ de las categorías, además contribuye en 2 de los 3 periodos establecidos; así pues es válido decir que ejerce una gestión focalizada en la modalidad Gestión de significados.

En el caso de E11, este no tiene un perfil estructural idóneo, pero las aportaciones realizadas en la gestión de la tarea académica lo catalogan para ejercer una gestión focalizada en la modalidad Tarea académica.

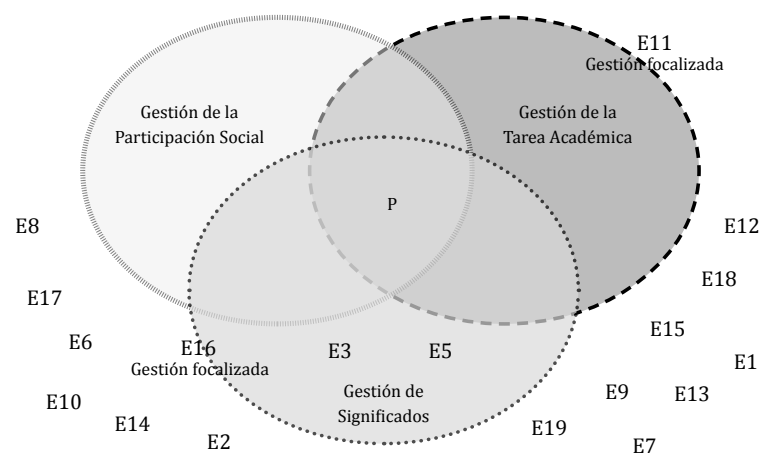

Figura 2. Mapa de distribución de la presencia docente (participantes portadores y no portadores)

Fuente: elaboración propia

En este marco, y como ha sido demostrado en las últimas décadas, existe un gran potencial de colaboración entre estudiantes universitarios para mejorar los procesos de construcción de conocimiento. Este potencial puede verse aumentado cuando la colaboración está mediada por tecnologías digitales, especialmente por redes asíncronas (Coll, Bustos y Engel, 2009). Sin embargo, concretar esas potencialidades no es tarea fácil, ya que no basta con poner a los estudiantes a trabajar en grupo, ni ofrecerles "tecnologías colaborativas potentes" (Lipponen y Lallimo, 2004, Citado en Coll y Monereo, 2008) para que emerjan los mecanismos y las pautas interactivas que explican la eficacia del aprendizaje colaborativo. Su aparición o no, y con ella la mayor o menor contribución de la colaboración entre estudiantes para el aprendizaje, depende en gran medida de la naturaleza de su implicación y participación en el trabajo grupal.

Los resultados obtenidos también permiten identificar opciones de mejora de la intervención propuesta y orientar algunas perspectivas para contribuir al uso real (no potencial) que ofrecen las redes asíncronas en la construcción de conocimiento a través de la colaboración y los mecanismos de influencia educativa. La primera de ellas tiene que ver con conseguir un mejor ajuste de la carga de trabajo y la exigencia a los estudiantes a lo largo del proceso, sin que ello afecte a la eficacia de la intervención. Asimismo, es importante atender de manera más equilibrada tanto a los componentes cognitivos como a los motivacionales y afectivos implicados en el desarrollo de la tarea, así como flexibilizar los apoyos ofrecidos para que se adapten mejor a grupos y estudiantes con experiencia y habilidades diversas de trabajo en equipo. Todo ello dirigido a tratar de apoyar de maneras cada vez más ajustadas la mejora de la construcción de conocimiento en este tipo de redes.

\section{Conclusiones}

A manera de cierre y con el propósito de contribuir al fortalecimiento de las prácticas educativas que se enmarcan bajo un enfoque socioconstructivista, en entornos virtuales de aprendizaje se presentan algunos aspectos que sobresalen a lo largo del análisis y se considera que pueden ser valiosos en la implementación de este tipo de experiencias educativas.

- Tanto la frecuencia como el patrón de acceso y participación (lectura y escritura) de docente y estudiantes favorecen los procesos de interlocución para el intercambio de conceptos, argumentos y otras formas de actuación que posteriormente se ven evidenciados en el análisis de contenido.

- El discurso -en este caso, escrito- permite la construcción de significados y atribución de sentido. De esta manera, los procesos de ayuda educativa se concretan a través de los intercambios comunicativos que se dan entre los participantes, particularmente en la construcción de significados compartidos.

- La aparición de diferentes modalidades (gestión de presencia docente, gestión de significado, gestión de la tarea académica, gestión de participación social) y grados (total, parcial, 
focalizada) de presencia docente, evidencian que esta no es ni debe ser patrimonio exclusivo del profesor.

- La enseñanza y el aprendizaje bajo un enfoque socioconstructivista en un ambiente virtual de aprendizaje favorecen la interacción y la distribución de la presencia docente. Esta se debe promover desde los aspectos cognitivos y afectivos, pues en el caso estudiado se privilegia de manera relevante la dimensión de la gestión de significados, lo que genera pocos espacios para promover las gestiones correspondientes a la parte afectiva y social, factores necesarios para la construcción de conocimiento en la actual sociedad de la información.

\section{Referencias}

Anderson, T. (2004). Teaching in an Online Learning Context. En T. Anderson, y E. Fathi, The theory and practice of online learning (pp. 271-293). Athabasca: Athabasca University.

Anderson, T., Rourke, L. y Garrison, R. (2001). Assessing teaching presence in a computer conferencing context. The Journal of Asynchronous Learning Networks, 5, 50-63.

Borgobello, A. y Roselli, N. (2016). Rendimiento académico e interacción sociocognitiva de estudiantes en un entorno virtual. Educação e Pesquisa, 42(2), 359-374.

Bustos, A. (2011). Presencia docente distribuida, influencia educativa y construcción del conocimiento en entornos de enseñanza y aprendizaje basados en la comunicación asíncrona escrita (tesis de doctorado). Universidad de Barcelona, España.

Bustos, A., Coll, C. y Engel, A. (2009). Presencia docente distribuida en redes asíncronas de aprendizaje. Definición teórica y perspectiva multimétodo para su estudio. En F. Díaz Barriga, G. Hernández y M. A. Rigo. Aprender y enseñar con Tic en educación superior: contribuciones del socioconstructivismo (pp. 97-128). México DF: Universidad Nacional Autónoma de México.

Camps, A. (2003). Texto, proceso, contexto, actividad discursiva: puntos de vista diversos sobre la actividad de aprender y de enseñar a escribir. En A. Camps, T. Colomer, C. Jany, J. Dolz, N. Farrera, y R. Fort, Serie didáctica de la lengua y la literatura (pp. 13-31). Barcelona: Graó.

Coll, C. (2009). Las fuentes de la concepción constructivista: principios básicos e ideas directivas. En C. Coll, Construcción de conocimiento en la escuela (p. 107). Barcelona: Uoc.
Coll, C. (2010). Enseñar y aprender, construir y compartir: procesos de aprendizaje y ayuda educativa. En C. Coll, Desarrollo, aprendizaje y enseñanza en la Educación Secundaria (pp. 31-61). Barcelona: Graó.

Coll, C. y Martí, E. (2001). La educación escolar ante las nuevas tecnologías de la información y la comunicación. En C. Coll, A. Marchesi y J. Palacios. Desarrollo psicológico y educación. Vol. II: Psicología de la educación escolar (pp.623-655). Madrid: Alianza.

Coll, C. y Monereo, C. (2008). Psicología de la educación virtual. Barcelona: Morata.

Coll, C., Bustos, A. y Engel, A. (2009). Perfiles de participación y presencia docente distribuida en redes asíncronas de aprendizaje: la articulación del análisis estructural y de contenido. Revista de Educación, 354, 657-688.

Coll, C., Colomina, R., Onrubia, J. y Rochera, M. (1992). Actividad conjunta y habla: una aproximación a los mecanismos de influencia educativa. Infancia $y$ Aprendizaje, 189-232.

Coll, C., Engel, A. y Niño, S. (2017). La actividad de los participantes como fuente de información para promover la colaboración. Una analítica del aprendizaje basada en el modelo de influencia educativa distribuida. RED. Revista de Educación a Distancia. 53, 36.

Coll, C., Onrubia, J. y Mauri, T. (2008). Ayudar a aprender en contextos educativos: el ejercicio de la influencia educativa y el análisis de la enseñanza. Revista de Educación, 346, 33-70.

Colomina, R. y Onrubia, J. (2001). Interacción educativa y aprendizaje escolar: la interacción entre alumnos. En C. Coll, A. Marchesi y J. Palacios. Psicología de la educación escolar (pp. 415-435). Madrid: Alianza.

Cuéllar, D., Bernal-Velasco, O., Pérez-Soto, J. y MontenegroAldana, I. (2012). Valoración de la presencia docente en Micea Virtual. Revista Memorias, 10(17), 113-124.

Galvis, Á. (2005). Criterios y escala de valoración TIGRE. Recuperado de http://aportetigre.blogspot.com/

García, B. y Pineda, O. (2010). La construcción de conocimiento en foros virtuales de discusión entre pares. Revista Mexicana de Investigación Educativa, 15(44), 85-111.

Garrison, D. y Anderson, T. (2005). El e-learning en el siglo XXI. Barcelona: Octaedro.

Gros, B. y Adrián, M. (2009). Estudio sobre el uso de los foros virtuales para favorecer las actividades colaborativas en la enseñanza superior. Teoría de la Educación: Educación y Cultura en la Sociedad de la Información. 5, 1-10. 
Gros, B. y García, I. (2013). Teoría de la educación. Educación y cultura en la sociedad de la información [Editorial]. 14(1), 4-9.

Guilleumas, R. (2015). Patrones de participación y nivel y modalidad de presencia docente distribuida en dos cursos en línea apoyados en el uso de foros asíncronos de discusión escrita (tesis de doctorado). Universidad de Salamanca, España.

Hernández-Sellés, N., González-Sanmamedy, M. y MuñozCarril, P. (2015). El rol docente en las ecologías de aprendizaje: análisis de una experiencia de aprendizaje colaborativo en entornos virtuales. Profesorado. Revista de Currículum y Formación de Profesorado, 19(2), 147-163.

Leal, D. (2011). Conectivismo y aprendizaje en red. [Video]. Recuperado de https://www.youtube.com/ watch?v=5Kpoo2vZkeQ

Lévy, P. (2004). Inteligencia colectiva: por una antropología del ciberespacio. La Habana: Organización Panamericana de la Salud.

Ministerio de Educación Nacional. (2010). Decreto 1295. Bogotá.
Onrubia, J. (1997). Aprendizaje y construcción de conocimientos en la educación secundaria obligatoria. Signos. Teoría y Práctica de la Educación, 20, 16-23.

Onrubia, J. (2005). Aprender y enseñar en entornos virtuales: actividad conjunta, ayuda pedagógica y construcción del conocimiento. RED. Revista de Educación a Distancia.

Palacios, J., Rodríguez, J. y Forero, D. (2015). Educación y campus virtual, nuevos escenarios de formación. Revista Científica, 22, 97-110.

Pérez, M. (2009). La comunicación y la interacción en contextos virtuales de aprendizaje. Revista Apertura, 1(1), 1-13.

Stake, R. (2005). Investigación con estudio de casos. Madrid: Morata.

Tobón, M., Arbeláez, M., Falcón, M. y Bedoya, J. (2010). La formación docente al incorporar las TIC en los procesos de enseñanza y aprendizaje. Pereira: Universidad Tecnológica de Pereira.

Zhu, E. (2006). Interaction and cognitive engagement: An analysis of four asynchronous online discussions. Instructional Science, 34, 451-480. 